\title{
Dupuytren's contracture following burn injury of the hand: A case report and review of literature
}

\author{
Chenicheri Balakrishnan MD, Kristoffer B Sugg MD, William Huettner MD, Payam Jarrahnejad MD
}

C Balakrishnan, KB Sugg, W Huettner, P Jarrahnejad. Dupuytren's contracture following burn injury of the hand: A case report and review of literature. Can J Plast Surg $2008 ; 16(1): 49-51$.

In burn patients, scar contractures adjacent to or across the joints lead to disabling deformities. In Dupuytren's disease, the proliferative process involves the fascia of the palm and fingers, resulting in disabling flexion contractures of the fingers and the palm. A single insult involving the hand or even a more proximal injury may lead to Dupuytren's disease.

\section{Une contracture de Dupuytren après une brûlure de la main : Rapport de cas et analyse bibliographique}

\begin{abstract}
Chez les patients brûlés, les contractures cicatricielles adjacentes aux articulations ou sur les articulations provoquent des malformations invalidantes. Dans le cas de la maladie de Dupuytren, le processus prolifératif touche le fascia de la paume et des doigts, ce qui entraîne des contractures invalidantes en flexion des doigts et de la paume. Une seule agression de la main ou même une lésion plus proximale peut provoquer la maladie de Dupuytren.
\end{abstract}

Key Words: Burn; Dupuytren's contracture; Dupuytren's disease

$\mathrm{D}_{\mathrm{i}}$ upuytren's disease is a benign fibroproliferative disorder involving the palmar and digital fascia; manifestation depends on genetic and environmental factors. Both familial and sporadic forms exist. Reports of earlier presentation is usually associated with a positive family history, although the disease most often affects people older than 50 years $(1,2)$. The palmar and digital contractures are progressive, and result in significant functional disability. Although other conditions such as diabetes, alcoholism, smoking, HIV infection and seizures have been suggested as associated factors, specific causal relationships are yet to be determined. No single causative factor has been described. We report a rare case of Dupuytren's contracture in a young man who developed the disorder following a burn injury, implicating burn as the pathophysiological factor.

\section{CASE PRESENTATION}

A 31-year-old right-hand-dominant man presented with a one-month history of limited extension of the ring and middle fingers of left hand. Initially, he was unable to fully extend his fingers and despite home stretching exercises, the condition was progressive. He had a history of superficial burns to the palm of his left hand from a barbeque approximately four months before presentation, which was treated in the emergency room. He was seen once in the burn outpatient clinic as a follow-up. The burn wound healed in approximately 10 days and, according to the patient, the hand was always hyperemic. He worked as a financial analyst. He had no family history of diabetes or Dupuytren's disease, and denied any past medical history, medications or alcohol.

Four months after the burn injury, physical examination revealed redness on the sides of the ring and long fingers with prominent palmar bands over the metacarpals extending to the proximal interphalangeal joints. He had an extension deficit of $15^{\circ}$ at the metacarpophalangeal joint compared with the contralateral hand (Figure 1). Night splinting for three months was deemed unsuccessful with no improvement in the contractures. The palmar bands were excised through a transverse palmar incision. The histopathological examination of the excised fascia showed fibromatous proliferation suggestive of Dupuytren's disease.

\section{DISCUSSION}

Dupuytren's disease is a disorder of the palmar and digital fascia that results in nodules and cords. Disabling contractures of the palm and digits are consequences of thickening and shortening of fibrous bands or cords during this fibroproliferative process. The first web space cord can cause an adduction contracture of the thumb. When the cords of scar tissue result in stiffness or contracture, the process is called Dupuytren's contracture. The disease is attributed to Guillaume Dupuytren, a French surgeon, because of his expertise on the clinical findings, pathogenesis, prognosis and treatment of the disease. He clarified the disease in 1831. Plater and Cline provided the earliest known records of the disease and its surgical treatment, respectively.

Dupuytren's disease belongs to the group of fibromatoses, which are classified as infantile and adult, and superficial (fascial) and deep (musculoaponeurotic). Dupuytren's disease falls in the adult superficial fibromatosis category as palmar fibromatosis, along with knuckle pads, plantar fibromatosis (Ledderhose disease) and penile fibromatosis (Peyronie's disease). The Dupuytren's diathesis is an aggressive variant of the disease with earlier presentation and more rapid progression 


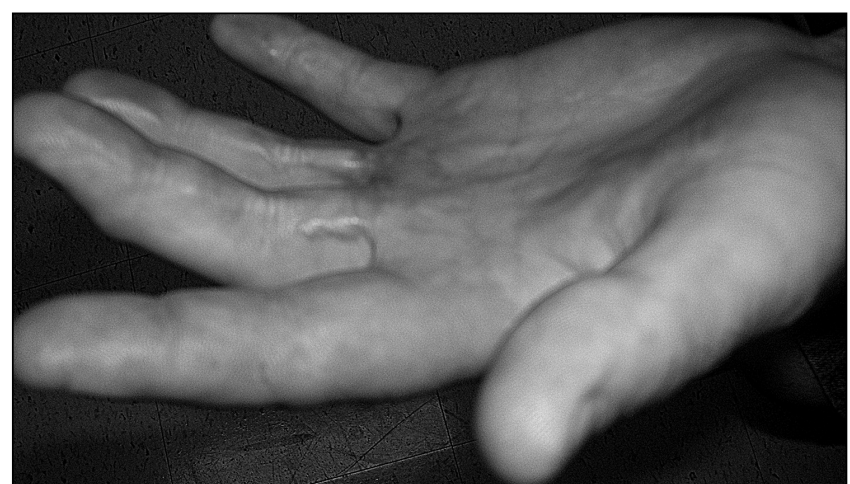

Figure 1) Dupuytren's bands in the palm causing contracture of the metacarpophalangeal joint in a patient following burn injury

comprised of Ledderhose and Peyronie's disease accompanied with knuckle pads of the dorsum of the proximal interphalangeal joints (Garrod's nodes) (1,2).

Dupuytren's disease may have originated among the Vikings of northern Europe. Elliot (3) examined the possible association of the disease with the Scottish bagpipe-playing MacCrimmon clan and the Papal Sign of Benediction. The pathological understanding, spontaneous rupture and the advent of surgical treatment of the disease in the late 18th and early 19th centuries in Europe and North America have been reviewed in detail $(3,4)$.

The incidence of Dupuytren's contracture is highest in Caucasians, historically those of Celtic descent. The prevalence of Dupuytren's disease in a Celtic family was 24\%, which increased to $74 \%$ with the inclusion of close relatives (1). The disease affects men seven to 15 times more often than it does women. Although the incidence of Dupuytren's disease is higher in men, the ratio between men and women may be smaller because the disease is less severe in women, and therefore, it may go unnoticed until later in life. The incidence becomes equal after 70 years of age. Because most cases of Dupuytren's disease occur in patients older than 50 years of age, one can expect an increasing incidence as the aging population increases. The ulnar rays are most commonly affectedthe ring, small and middle fingers, respectively. The incidence of Dupuytren's disease also increases with diabetes, smoking, chronic alcoholism, epilepsy and certain infections. Approximately 5\% of individuals with Dupuytren's disease are diabetic, with an increased prevalence proportional to the duration of the diabetes. One group (1) showed a higher incidence of retinopathy and Dupuytren's contracture, and attributes the cause to microangiopathic changes. Similarly, smoking is associated with microvascular changes that may contribute to Dupuytren's disease (1). The prevalence of Dupuytren's disease in those with chronic alcoholism increases in proportion to the amount of alcohol consumed (5). Injury to the distal radius (6), burns (7) and even shoulder dislocation (8) have been reported to trigger the onset of Dupuytren's contracture.

Various conditions seem to be responsible for proliferation of fibroblasts and collagen deposition, leading to contracture along the lines of stress. Although the incidence of Dupuytren's disease is two to three times higher in individuals with epilepsy, opinions about the cause differ. Studies have shown electroencephalogram abnormalities were more common in patients with Dupuytren's disease than in others, and a correlation between increased amounts of barbiturate medication with higher occurrence of Dupuytren's disease (9. 11). Rheumatoid arthritis is associated with a decreased incidence of Dupuytren's contracture (12).

Dupuytren's contracture is associated with thickening and nodularity of the palmar fascia. All the digits, including the thumb, can be affected. Most commonly, the contracture of the palmar fascia causes the ring and little fingers to bend into the palm, with inability to extend the fingers. Although results of previous studies that linked repetitive manual labour to Dupuytren's disease were inconclusive, more recent studies show that the incidence of the disease is 5.5 times higher with such labour. Anecdotal evidence suggests that Dupuytren's disease occurs after trauma. Regardless, a positive family history may play a role in both occupational and traumatic cases of Dupuytren's disease. The present status of adjudication for workers claiming compensation for Dupuytren's disease is inconsistent and, therefore, unfair to both workers and employers. In some eastern European countries, Dupuytren's disease is classified as an industrial disease, whereas in other countries it is considered to have no relation to manual work or hand injury. In jurisdictions that sometimes award compensation, the reasons for acceptance or rejection of a claim vary from case to case and are not necessarily based on our present knowledge of the disease (13).

The disease has long been known to have an autosomal dominant mode of inheritance with variable penetrance. Neumuller et al (14) demonstrated an increased relative risk of 2.94 for individuals who express human leukocyte antigenDR3. A number of growth factors, immunological mediators and free radicals are implicated in the development of Dupuytren's contractures. Most likely, an inciting disease or event in a genetically predisposed individual causes a cascade of events that may include processes that promote the formation of growth factors and free radicals, which ultimately leads to abnormal fibroproliferation and the appearance of the characteristic nodule. When homeostasis is ultimately achieved and when fibroblastic growth lessens, the pathological nodule and cord remain. The striking variation in prevalence, approaching $30 \%$ in individuals over the age of 60 years in Norway, is strong evidence for an inherited susceptibility to Dupuytren's disease (9). It remains unclear whether the many apparently sporadic cases have a genetic basis. The inherited susceptibility may influence tissue's sensitivity to the effects of environmental exposures. High prevalence, late age of onset and the possibility of nongenetic cases are features of Dupuytren's disease that complicate genetic studies, as can be seen from estimates of relative risk. Identification of susceptibility loci is a worthwhile goal becasue it may throw entirely new light on the nature of Dupuytren's disease and ultimately may open avenues to new treatments. (9).

Histological examination of the excised tissues from various sites showed the presence of myofibroblasts only in the Dupuytren's nodule. These myofibroblasts have been implicated in the disease (15). In the palm, contractures occur in the pretendinous bands and natatory ligaments, which are subsequently called the pretendinous cord and natatory cord, respectively. In addition, attachment of the transverse fibres of the palmar aponeurosis, found at the crease between the index finger and the thumb, may also form a contracture. In the digits, normal fascial structures, including the volar 
superficial fascia and lateral digital sheets, effectively become the central cord and lateral cords, respectively (1).

The present patient, who had no predisposition to the disease, developed pretendinous bands in the palm four months following injury. Because this was progressive, he underwent excision of the fascia. Histology confirmed this to be fibromatous process of Dupuytren's disease.

\section{REFERENCES}

1. Murrell GA, Hueston JT. Aetiology of Dupuytren's contracture. Aust N Z J Surg 1990;60:247-52.

2. Watson KH, Paul H Jr. Pathologic anatomy. Hand Clin 1991;7:661-8.

3. Elliot D. The early history of Dupuytren's disease. Hand Clin 1999;15:1-19.

4. Whaley DC, Elliot D. Dupuytren's disease: A legacy of the north? J Hand Surg [Br] 1993;18:363-7.

5. Bradlow A, Mowat AG. Dupuytren's contracture and alcohol. Ann Rheum Dis 1986;45:304-7.

6. Kelly SA, Burke FD, Elliot D. Injury to the distal radius as a trigger to the onset of Dupuytren's disease. J Hand Surg [Br] 1992;17:225-9.
7. Balakrishnan C, Emanuele JA, Chow IJ. Dupuytren's contracture after burns of the upper extremity. J Burn Care Rehabil 1997; 18:245-6.

8. Hueston JT. Dupuytren's contracture and specific injury. Med J Aust 1968;1:1084-5.

9. Burge P. Genetics of Dupuytren's disease. Hand Clin 1999;15:63-71.

10. Bayat A, Watson JS, Stanley JK, et a. Genetic susceptibility in Dupuytren's disease. TGF-betal polymorphisms and Dupuytren's disease. J Bone Joint Surg Br 2002;84:211-5.

11. Gordon SD. Dupuytren's contracture: Heredity as an etiological factor. Can J Surg 1959;2:421.

12. Arafa M, Steingold RF, Noble J. The incidence of Dupuytren's disease in patients with rheumatoid arthritis. J Hand Surg [Br] 1984;9:165-6.

13. McFarlane RM. Dupuytren's disease: Relation to work and injury. J Hand Surg [Am] 1991;16:775-9.

14. Neumuller J, Tohidast-Akrad M, Ammer K, et al. Ultrastructural and autoradiographic investigations of cell cultures derived from tendons or ligamentous material from patients with fibromatous disorders. Exp Cell Biol 1988;56:113-30.

15. Hueston JT, Hurley JV, Whittingham S. The contracting fibroblast as a clue to Dupuytren's contracture. Hand 1976;8:10-2. 